\title{
Protective effects of diltiazem and tadalafil on shock wave- -induced kidney injury in rats
}

\author{
Ozmerdiven $\mathrm{G}^{1}$, Vuruskan $\mathrm{BA}^{2}$, Kaygisiz $\mathrm{O}^{1}$, Vuruskan $\mathrm{H}^{1}$ \\ Department of Urology, Uludag University, School of Medicine, Bursa, Turkey. \\ gozmerdiven@gmail.com
}

\begin{abstract}
BACKGROUND: We aimed to compare the protective effects of tadalafil and diltiazem on renal histology after ischemia and reperfusion injury in a rat model of shock wave lithotripsy.

METHODS: A total of 40 adult, male Sprague-Dawley rats were randomized into four groups as follows; control group (group C), group S (SWL + nephrectomy), group $T$ (SWL + tadalafil given before nephrectomy) and group D (SWL + diltiazem given before nephrectomy). Both kidneys were evaluated regarding tubular damage, peritubular fibrosis and heat shock protein-70 (HSP-70) immune-expression of glomeruli, cortical and medullar collector tubules on light microscopy.

RESULTS: HSP-70 levels of cortical and medullar collector tubules, tubular damage and peritubular fibrosis scores were decreased in group T compared with group S. Similarly, HSP-70 immunostaining levels on cortical and medullar collector tubules, tubular damage and peritubular fibrosis scores were decreased in group D compared with group S. No significant difference was detected between group D and group $\mathrm{T}$ for all parameters. CONCLUSION: As a result, shock waves induced renal cell damage due to increment of HSP-70 levels, morphological irregularity in tubules and increased peritubular fibrosis. Tadalafil and diltiazem had beneficial effects in decreasing renal tissue damage which was caused by SWL (Tab. 2, Fig. 6, Ref. 29). Text in PDF www.elis.sk. KEY WORDS: diltiazem, ischemia-reperfusion injury, heat shock protein, shock wave lithotripsy, renal tubules, tadalafil.
\end{abstract}

\section{Introduction}

Shock wave lithotripsy (SWL) has been acknowledged as an effective and non-invasive treatment modality for urolithiasis and is the first choice of treatment for most patients with urolithiasis as it is the least invasive method (1). However, it is associated with both short- and long-term risks of renal and extra renal complications (1-3). Several mechanisms, such as cavitation bubbles, temporary decrease of renal perfusion and ischemia-reperfusion (I/R) injury, are responsible for renal injury during SWL (4-6). New generation SWL devices are clinically used to decrease complication rates (1). Furthermore, several molecules including calcium channel blockers (CCBs), anti-inflammatory agents, phosphodiesterase type 5 (PDE5) inhibitors and antioxidants, have been shown to reduce I/R injury and SWL complications (7-16).

The use of CCBs in I/R injury decreases calcium influx through the cell membrane and reduces phospholipase activity, thereby protecting against tubular injury by reducing levels (12). A previous study reported the protective effect of diltiazem through the

${ }^{1}$ Department of Urology, Uludag University, School of Medicine, Bursa, Turkey, and ${ }^{2}$ Department of Pathology, Uludag University, School of Medicine, Bursa, Turkey

Address for correspondence: G. Ozmerdiven, Dr, Department of Urology, Uludag University, School of Medicine 16059 Gorukle, Bursa, Turkey. Phone: +90.5353586163 reduced calcium transportation and phospholipase activation in SWL-induced kidney damage (13).

Another group of molecules, PDE5 inhibitors, are currently in clinical use for erectile dysfunction and benign prostatic hyperplasia. These drugs are specific for the hydrolysis of cyclic guanosine monophosphate (cGMP) and play an important role in the regulation of nitric oxide (NO) release (14). Tadalafil, a PDE5 inhibitor exhibiting a maximum plasma concentration higher than other PDE5 inhibitors, has been shown to have protective effects in studies of renal $\mathrm{I} / \mathrm{R}$ injury $(15,16)$.

In our study, we aimed to assess the histopathological findings of renal trauma and inflammation due to SWL exposure and compared the effects of tadalafil and diltiazem to prevent renal $\mathrm{I} / \mathrm{R}$ injury in a rat model.

\section{Materials and methods}

All experimental studies were performed in compliance with ethical guidelines and confirmed by the local Ethics Committee of Uludag University (2015-02/05). Forty Sprague-Dawley rats were observed in climate controlled chambers. Surgical and SWL procedures were systematically and appropriately performed.

\section{Surgical and SWL Procedure}

The rats were anaesthetised via an intramuscular injection of $1 \mathrm{mg} / \mathrm{kg}$ ketamine $\mathrm{HCL}$ and $10 \mathrm{mg} / \mathrm{kg}$ xylazine $\mathrm{HCl}$. Laparotomy 
Tab. 1. Comparison of groups between each other regarding the tubular damage and peritubular fibrosis scores. Numbers indicate the p values of Mann-Whitney U test which was utilized to compare two individual groups.

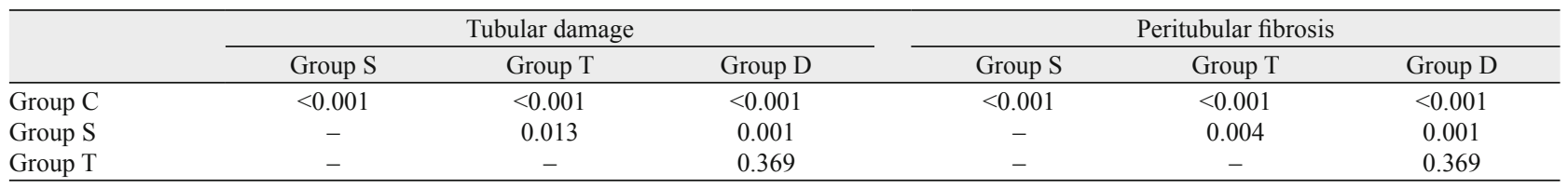

Tab. 2. Comparison of groups between each other regarding HSP-70 staining of glomeruli, cortical and medullar collector tubules. Numbers indicate the p values of Mann-Whitney $U$ test which was utilized to compare two individual groups.

\begin{tabular}{|c|c|c|c|c|c|c|c|c|c|}
\hline & \multicolumn{3}{|c|}{ Glomeruli } & \multicolumn{3}{|c|}{ Cortical collector tubules } & \multicolumn{3}{|c|}{ Medullar collector tubules } \\
\hline & Group S & Group T & Group D & Group S & Group T & Group D & GroupS & Group T & Group D \\
\hline Group C & $<0.001$ & $<0.001$ & $<0.001$ & $<0.001$ & $<0.001$ & $<0.001$ & $<0.001$ & $<0.001$ & $<0.001$ \\
\hline Group S & - & 0.314 & 0.512 & - & 0.002 & 0.001 & - & 0.005 & 0.001 \\
\hline Group T & - & - & 0.678 & - & - & 0.799 & - & - & 0.718 \\
\hline
\end{tabular}

was performed in all rats. Following abdominal midline incision, two haemoclips were attached to the perirenal fatty tissue. All animals except those in control group, were underwent SWL after laparotomy. Both haemoclips were targeted during the SWL procedure. In a single session, a total of 1500 shock waves were applied to each kidney using the Multimed Classic ${ }^{\circledR}$ (Elmed Inc., 2006, Ankara, Turkey) lithotripsy system at an energy setting of $14 \mathrm{kV}$.

\section{Groups}

A total of 40 male Sprague-Dawley rats were randomized into four groups of 10 rats. In the control group (group C), only laparotomy and metal clipping was performed. In group $\mathrm{S}$, the rats received no treatment before the SWL procedure. In the tadalafil treatment group (group T), tadalafil (Cialis, Lilly, USA) was dissolved in saline solution and administrated as a single dose (1 mg/ $\mathrm{kg}$ ) through an orogastric tube $60 \mathrm{~min}$ before the SWL procedure. In the diltiazem treatment group (group D), diltiazem (Diltizem, Mustafa Nevzat Pharmacy, Turkey) was dissolved in saline solution and administrated as a single dose $(10 \mathrm{mg} / \mathrm{kg})$ through an orogastric tube $60 \mathrm{~min}$ before the SWL procedure. All subjects had bilateral nephrectomy 7 days after the procedure

\section{Histopathological examination}

The histopathological examination included a microscopic evaluation of glomeruli and tubular morphology. For this purpose 100 tubules and 20 glomeruli were evaluated randomly and were scored for each section. The morphology of tubules was classified into the four grades based on extent of tubular damage: grade 0 (normal tubule and glomeruli), 1 (mild abnormality in tubules, $<$ $50 \%$ ), 2 (moderate abnormality in tubules, 50-90\%) and 3 (most tubules were losts, $90-100 \%$ ). Peritubular fibrosis score was also classified into flowing four grades: grade 0 (no fibrosis), $1(<50$ $\%$ peritubular fibrosis), 2 (50-90\% peritubular fibrosis) and 3 (90-10 0\% peritubular fibrosis).

Heat shock protein (HSP), a well-known intracellular stress protein, has been reported as an indicator of thermal and oxidative stress (17). First, the paraffinised tissues were deparaffinised with using xylene. Endogenous peroxidase in tissues was deactivated with $1 \% \mathrm{H}_{2} \mathrm{O}_{2}$ solution for $5 \mathrm{~min}$. The tissues were prepared with
$10 \mu \mathrm{M}$ sodium citrate solution and placed in a microwave, and primary antibody (hsp70Ab.2, Clone W27; Neo Markers) was applied to the tissues. HSP-70 staining was detected using the LSAB 2 kit and DAB chromogen (DAKO). Glomeruli and tubular staining was compared among all groups. HSP-70 staining level was recorded as 0 (non-staining), 1 ( $<5 \%$ stained), 2 (5-50\% stained) and 3 (> $50 \%$ stained).

\section{Statistical analysis}

Statistical analysis was performed using the SPSS software ver. 22.0 (IBM Corporation, Chicago, IL). The non-parametric Kruskal-Wallis test was used to determine the statistically significant differences among the groups. The Mann-Whitney U test was used to compare the differences among the groups. A p value of $<0.05$ was considered as statistically significant.

\section{Results}

Renal tubular damage and peritubular fibrosis were significantly increased in group $\mathrm{S}$ compared with those in the control group $(p<0.001)$. Tubular damage was significantly increased in group $\mathrm{S}$ compared with that in groups $\mathrm{T}$ and $\mathrm{D}(\mathrm{p}=0.013, \mathrm{p}=0.001$, respectively). Peritubular fibrosis was significantly decreased in groups $\mathrm{T}$ and $\mathrm{D}$ compared with that in group $\mathrm{S}(\mathrm{p}=0.004, \mathrm{p}=$ 0.001 , respectively). No significantly difference was detected between the drug-receiving groups (Tab. 1).

The HSP-70 staining intensity in the glomeruli was higher in group $\mathrm{S}, \mathrm{T}$ and $\mathrm{D}$ than in the control group; however differences in the HSP-70 staining intensities in the glomeruli among groups $\mathrm{S}, \mathrm{T}$ and D were statistically insignificant (Table II). Proximal tubule cells were not stained with HSP-70 in any group.

The HSP-70 staining intensity in cortical and medullar collector tubules was significantly higher in group $\mathrm{S}$ than control group $(\mathrm{p}<0.001)$. HSP-70 staining of medullar collector tubules was higher in group $\mathrm{S}$ than in groups $\mathrm{T}$ and $\mathrm{D}(\mathrm{p}=0.005$ and $\mathrm{p}=$ 0.001 respectively). Similarly HSP-70 staining of cortical collector tubules was higher in group $\mathrm{S}$ than in groups $\mathrm{T}$ and $\mathrm{D}(\mathrm{p}=0.002$ and $p=0.001$ respectively). Moreover, no statistically significant difference was detected between groups $\mathrm{D}$ and $\mathrm{T}$ (Tab. 2). 


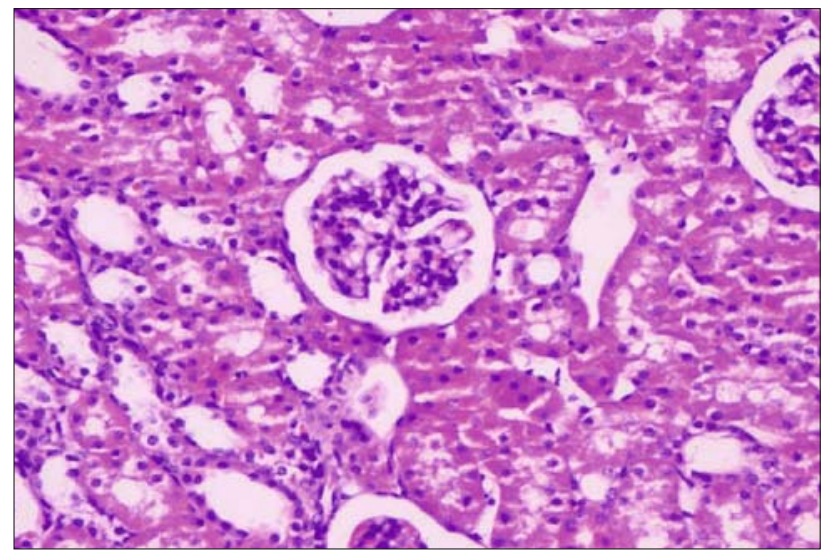

Fig. 1. Regular morphology of renal parenchyma in control group (Hematoxylin-Eosin x200).

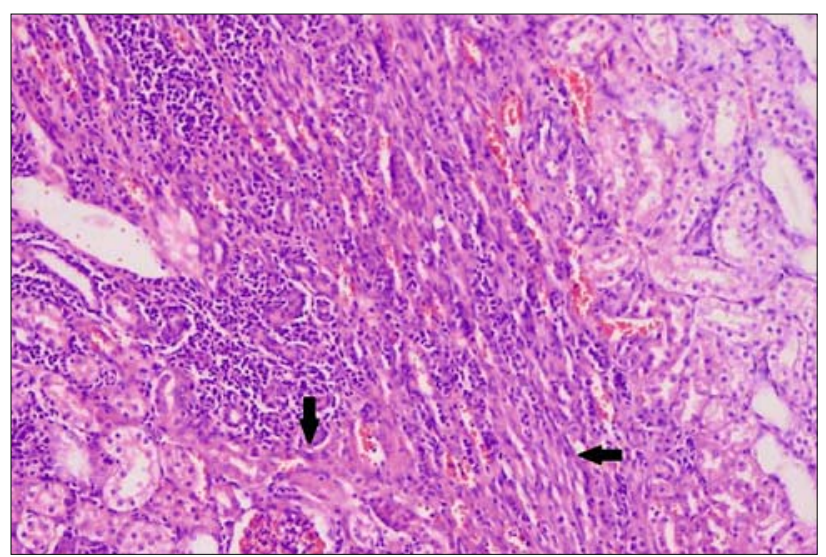

Fig. 2. Tubular necrosis and peritubular fibrosis in group S (Hematoxylin-Eosin 100).

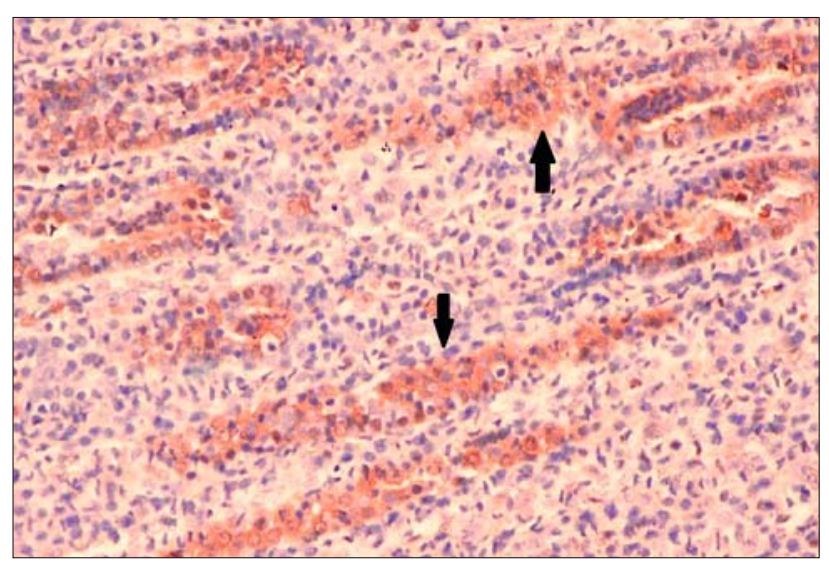

Fig. 3. HSP-70 staining of collector tubules in group S (HSP-70 x100).

Microscopic appearance was normal in the control group (Fig. 1). In group S, Bowman's space was enlarged, and most microvilli were lost at the epithelium of the proximal tubules. In cortical and medullar regions, tubular necrosis with hyaline degeneration was noticed. Interstitial space was enlarged, which was correlated with the presence of fibrosis (black arrows, Fig. 2). HSP-70 stain-

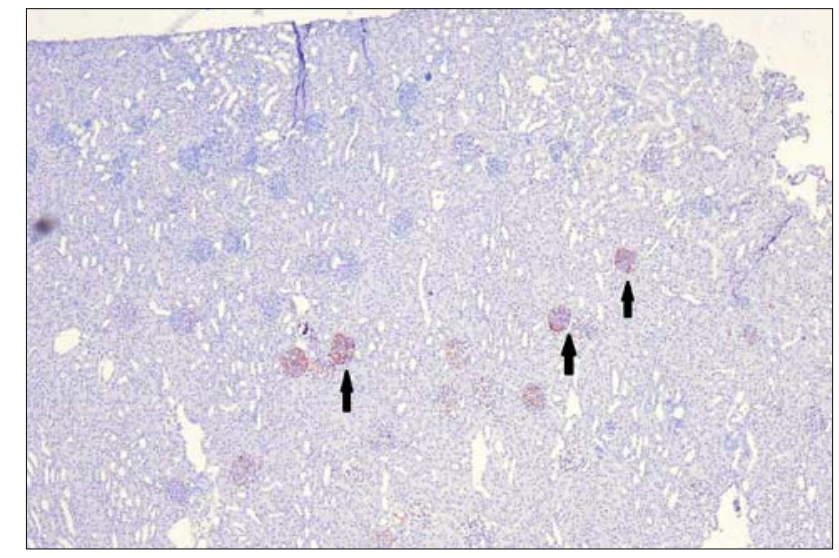

Fig. 4. HSP-70 staining of renal glomerulus in group T (HSP-70 x40).

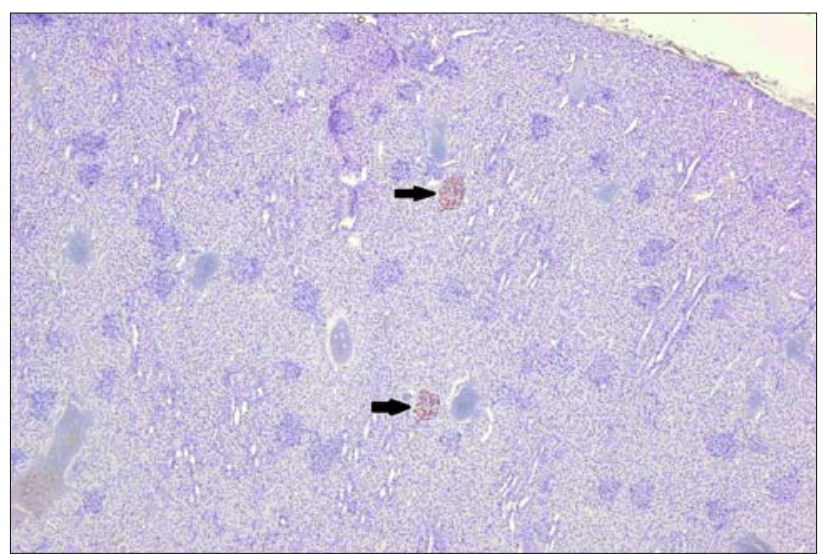

Fig. 5. HSP-70 staining of renal glomerulus in group D (HSP-70 x40).

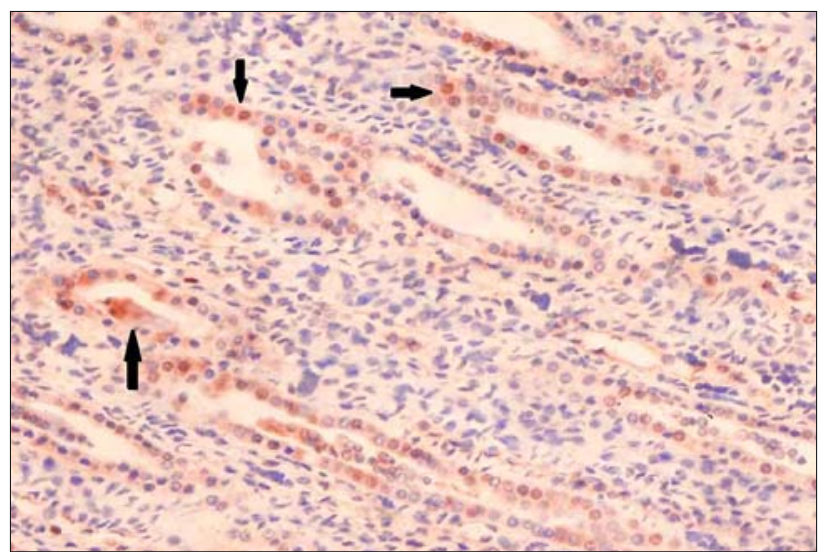

Fig. 6. HSP-70 staining of cortical collector tubules in group D (HSP$70 \times 100)$.

ing of tubular tissue was increased in group S (black arrows, Fig. 3). In group $T$, the morphology of capillaries in glomeruli and tubular cells was revealed to be normal. Areas with interstitial oedema were commonly observed near blood vessels. HSP-70 immunostaining of glomerular tissue was increased in group $\mathrm{T}$ (black arrows, Fig. 4). In group D, the kidney exhibited a pre- 
served regular morphology of glomeruli and tubular cells. Microvilli structures were found to be preserved in many epithelial cells lining the proximal tubules. In group D, HSP-70 immunostaining of glomerular tissue was increased (black arrows, Fig. 5), and HSP-70 staining of cortical collector tubules was decreased (black arrows, Fig. 6).

\section{Discussion}

SWL is a non-invasive treatment that is commonly used for treating urolithiasis. Although the side effects of SWL can be changed by modifying the device properties, such as shock power and number of shocks. SWL mostly causes vascular trauma and I/R injury. These effects of SWL lead to intraparenchymal haemorrage, migration of inflammatory cells and cytokines, fibrosis and loss of microvilli on tubules (18-20). Additionally, the presence of renal tubular enzymes offer further evidence of tubular injury (20).

CCBs are used in decreasing blood pressure by vascular vasodilation and decreasing peripheral resistance (22). In studies, these drugs have been shown to have beneficial effects on ischemia induced tissues $(22,23)$. These drugs effectively decrease blood pressure mainly through vasodilation and the reduction of peripheral resistance. During an ischemia period, dysfunction of the $\mathrm{Na}^{+}-\mathrm{Ca}^{+}$exchanger leads to an increase in calcium molecules. Subsequently, this intracellular calcium overload activates phospholipases, which occur membrane damage in I/R injury (23).

The effect of CCB on SWL-induced kidney damage was studied in literature. Li et al examined the beneficial effects of nifedipine (a CCB) and allopurinol (a xanthine oxidase inhibitor) on SWL-induced renal injury. The authors reported that patients treated with nifedipine had better results than those not receiving any drugs and there was no difference between the two drugs. They considered that these medicines reduced the effects of the reactive oxygen species on tissues (24). Strohmeier et al studied the effects of verapamil (a CCB) and nifedipine on SWL-treated patients and evaluated the tubular excretion markers after SWL treatment which indicated that these CCBs had protective effects on tubular injury and dysfunction of tubular excretion $(5,25)$.

In the present study, we preferred to use diltiazem for reducing SWL-induced renal injury, because of the observed beneficial effects of diltiazem in patients undergoing renal transplant surgery. In such cases, diltiazem is given intraoperativelly before removal of arterial clamp in our clinic. Before this study, the effect of diltiazem on HSP-70 levels on the SWL-induced kidney was not published in literature.

PDE5 inhibitors are currently in clinical use for erectile dysfunction, pulmonary hypertension and benign prostate hyperplasia $(14,26,27)$. These drugs hydrolyse cGMP and increase NO. NO regulates intracellular calcium levels, induces vasodilatation and improves endothelial dysfunction in I/R injury (28). Gasanov et al demonstrated that a single dose of tadalafil administrated before $\mathrm{I} / \mathrm{R}$ injury had protective effects against oxidative stress. Renal tubular damage and necrosis were found to be reduced in the microscopic examination of the tadalafil administration group (15).
HSP-70 has been correlated with cytoprotection in response to several injuries, including those due to oxidative stress and ischemia. When renal epithelial cells are injured, changes were observed in the HSP-70 protein structure and HSP-70 mRNA was increased (17). Danisoglu et al reported that tadalafil administration before SWL decreased HSP-70 levels in the SWL-induced kidney, however no difference was observed in HSP-70 staining between 3rd and 7th. days after SWL in the tadalafil treated rats. The authors suggested that renal damage from 3rd to 7 th days was continuous and did not decrease (10). HSP-72, another heat shock protein, participates in the molecular protein mechanism and also prevents cellular damage against apoptosis molecules, such as cytochrome-c in mitochondrial membrane injury. In a study tubular damage and renal dysfunction were found to be reduced by HSP-72 in I/R injury of the kidney (29).

\section{Conclusion}

The present study revealed that SWL enhanced renal damage in rats. Tadalafil and diltiazem result in reduced HSP-70 staining with better preservation of the renal histology, as well as protective effects on the SWL induced-kidney. On the other hand, no significant differences were observed in treatment groups. The differences between these drugs are in terms of selectivity, side effects and pharmacokinetic features in the body. The choice of the treatment should be made considering the clinical findings and concomitant diseases in the patients.

\section{References}

1. Argyropoulos AN, Tolley DA. Optimizing shock wave lithotripsy in the 21 st century. Eur Urol 2007; 52 (2): 344-352.

2. Shao Y, Connors BA, Evan AP, Willis LR, Lifshitz DA, Lingeman JE. Morphological changes induced in the pig kidney by extracorporeal shock wave lithotripsy: nephron injury. Anat Rec 2003; 275 (1): 979-989.

3. Skolarikos A, Alivizatos G, de la Rosette J. Extracorporeal shock wave lithotripsy 25 years later: complications and their prevention. Eur Urol 2006; 50 (5): 981-990.

4. Aksoy Y, Malkoc I, Atmaca AF, Aksoy H, Altinkaynak K, Akcay F. The effects of extracorporeal shock wave lithotripsy on antioxidant enzymes in erythrocytes. Cell Biochem Funct 2006; 24 (5): 467-469.

5. Strohmaier WL, Bichler KH, Koch J, Balk N, Wilbert DM. Protective effect of verapamil on shock wave induced renal tubular dysfunction. J Urol 1993; 150 (1): 27-29.

6. Munver R, Delvecchio FC, Kuo RL, Brown SA, Zhong P, Preminger GM. In vivo assessment of free radical activity during shock wave lithotripsy using a microdialysis system: the renoprotective action of allopurinol. J Urol 2002; 167 (1): 327-334.

7. Esrefoglu M, Tok OE, Aydın MS, Iraz M, Ozer OF, Selek S, Iraz M. Effect of beta-glucanon protection young and aged rats from renal ischmia and reperfusion injury. Bratisl Lek Listy 2016; 117 (9): 530-538.

8. Bas M, Tugcu V, Kemahli E, Ozbek E, Uhri M, Altug T et al. Curcumin prevents shock-wave lithotripsy-induced renal injury through inhibition of nuclear factor kappa-B and inducible nitric oxide synthase activity in rats. Urol Res 2009; 37 (3): 159-164. 
9. Ibrahim MY, Aziz NM, Kamel MY, Rifaai RA. Sodium hydrosulphide against renal ischmia/reperfusion and possible contribution of nitric oxide in adult male Albino rats. Bratisl Lek Listy 2015; 116 (11): 681-688.

10. Danisoglu M, Aytac B, Kilicaslan H, Dogan S, Vuruskan H. Reduction of shock wave lithotripsy-induced renal tubular injury by tadalafil. Bratisl Lek Listy 2013; 114 (11): 616-620.

11. Canbek M, Bayramoglu G, Senturk H et al. The examination of protective effects of gallic acid against damage of oxidative stres during induced-experimental renal ischemia-reperfusion in experiment. Bratisl Lek Listy 2014; 115 (9): 557-562.

12. Schrier RW, Burke TJ. Role of calcium-channel blockers in preventing acute and chronic renal injury. J Cardiovasc Pharmacol 1991; 18: 38-43.

13. Jan CR, Chen WC, Wu SN, Tseng CJ. Nifedipine, verapamil and diltiazem block shock-wave-induced rises in cytosolic calcium in MDCK cells. Chin J Physiol 1998 Dec 31; 41 (4): 181-188.

14. Lau LC, Adaikan PG. Mechanisms of direct relaxant effect of sildenafil, tadalafil and vardenafil on corpus cavernous. Eur J Pharmacol 2006; 541 (3): 184-190.

15. Gasanov F, Aytac B, Vuruskan H. The effects of tadalafil on renal ischemia reperfusion injury: an experimental study. Bosn J Basic Med Sci 2011; 11 (3): 158-162.

16. Guzeloglu M, Yalcinkaya F, Atmaca S, Bagriyanik A, Oktar S, Yuksel $\mathrm{O}$ et al. The beneficial effects of tadalafil on renal ischemia-reperfusion injury in rats. Urol Int 2011; 86 (2): 197-203.

17. Aufricht C. Heat-shock protein 70: molecular supertool? Pediatr Nephrol 2005; 20 (6): 707-713.

18. Evan AP, McAteer JA. Q-effects of shock wave lithotripsy. 549-570. In: Coe FL, Favus MJ, Pak CYC, Parks JH, Preminger GM (Eds). Kidney Stones: Medical and Surgical Management. Philadelphia: LippincottRaven; 1996.

19. Lien YH, Lai LW, Silva AL. Pathogenesis of renal ischemia/ reperfusion injury: lessons from knockout mice. Life Sci 2003; 74: 543-552.
20. Kohrmann KU, Back W, Benseman J, Florian J, Weber A, Kahmann $\mathbf{F}$ et al. The isolated perfosed kidney of the pig: new model to evaluate shock wave-induced lesions. J Endourol 1994; 8 (2): 105-110.

21. Willams P, Lopez H, Britt D, Chan C, Ezrin A, Hottendorf R. Characterization of renal ischemia-reperfusion injury in rats. J Pharmacol Toxicol Methods 1997; 37 (1): 1-7.

22. Josephson RA, Silverman HS, Lakatta EG, Stern MD, Zweier JL. Study of the mechanisms of hydrogen peroxide and hydroxyl free radicalinduced cellular injury and calcium overload in cardiac myocytes. J Biol Chem 1991; 266 (4): 2354-2361.

23. Burke TJ, Arnold PE, Gordon JA, Bulger RE, Dobyan DC, Schrier RW. Protective effect of intrarenal calcium membrane blockers before or after renal ischemia. Functional, morphological and mitochondrial studies. J Clin Invest 1984; 74 (5): 1830-1841.

24. Li B, Zhou W, Li P. Protective effects of nifedipine and allopurinol on high energy shock wave induced changes on renal function. J Urol 1995; 153: 596-598.

25. Strohmaier WL, Koch J, Balk N, Wilbert DM, Bichler KH. Limitation of shock-wave-induced renal tubular dysfunction by nifedipine. Eur Urol 1994; 25 (2): 99-104.

26. de Carvalho AC, Hovnanian AL, Fernandes CJ, Lapa M, Jardim C, Souza R. Tadalafil as treatment for idiopathic pulmonary arterial hypertension. Arq Bras Cardiol 2006; 87 (5): 195-197.

27. van Driel MF. Phosphodiesterase inhibitors: effectiveness and new applications. Ned Tijdschr Geneeskd 2006; 150 (29): 1613-1616.

28. Kukreja RC, Ockaili R, Salloum F, Yin C, Hawkins J, Das A et al. Cardioprotection with phosphodiesterase-5 inhibition novel preconditioning strategy. J Mol Cell Cardiol 2004; 36 (2): 165-173.

29. Zhang B, Rong R, Li H, Peng X, Xiong L Wang Y et al. Heat shock protein 72 suppresses apoptosis by increasing the stability of $\mathrm{X}$-linked inhibitor of apoptosis protein in renal ischemia/reperfusion injury. Mol Med Rep 2015; 11 (3): 1793-1799. 